\title{
BMJ Open Modified effect of active or passive smoking on the association between age and abdominal aortic calcification: a nationally representative cross- sectional study
}

\author{
Luyan Lv, ${ }^{1}$ Shixian $\mathrm{Wu},{ }^{2}$ Yungui Yang, ${ }^{1}$ Xiongli Yue ${ }^{1}{ }^{1}$
}

To cite: Lv L, Wu S, Yang Y, et al. Modified effect of active or passive smoking on the association between age and abdominal aortic calcification: a nationally representative crosssectional study. BMJ Open 2021;11:e047645. doi:10.1136/ bmjopen-2020-047645

- Prepublication history and additional supplemental material for this paper are available online. To view these files, please visit the journal online (http://dx.doi.org/10.1136/ bmjopen-2020-047645).

$\mathrm{LL}$ and SW contributed equally.

Received 08 December 2020 Accepted 24 September 2021

Check for updates

(C) Author(s) (or their employer(s)) 2021. Re-use permitted under CC BY. Published by BMJ.

${ }^{1}$ Department of Geriatrics, Qujing First People's Hospital, Qujing, Yunnan, China

${ }^{2}$ Ministry of Science and education, Qujing First People's Hospital, Qujing, Yunnan, China

\section{Correspondence to}

Dr Xiongli Yue;

yxl13529891626@163.com and

Dr Yungui Yang;

1369687326@qq.com

\section{ABSTRACT}

Objective The deleterious effects of smoking on atherosclerosis were well known; however, the interaction among ageing, smoking and atherosclerosis remains unclear. This study tested the hypothesis that the association between age and vascular calcification, a critical mark of atherosclerosis, was modified by smoking.

Design Cross-sectional study.

Setting A nationally representative sample, the National Health and Nutrition Examination Surveys 2013-2014. Participants This study included 3140 adults aged $40-80$ years with eligible data for abdominal aortic calcification (AAC). Active and passive smoking exposure was identified through self-reports and tobacco metabolites (serum cotinine and urinary 4-methylnitrosamino-3-pyridyl-1butanol).

Primary outcome measures AAC score was determined using dual-energy X-ray absorptiometry (DXA) scans. OR was estimated using the logistic regression method to assess the association between age and the presence of severe or subclinical AAC stratified by smoking exposure. The survey-weighted Wald test was used to evaluate potential interactions.

Results AAC was positively associated with age in the general population. After adjustment for age, sex, race/ ethnicity and other cardiovascular risk factors, age was significantly associated with the odds of severe AAC (OR for each 5-year increase in age: $1.66,95 \% \mathrm{Cl} 1.48$ to $1.87, p<0.001)$. As expected, the association between age and vascular calcification was especially stronger in smokers than in never smokers ( $p$ value for interaction $\leq 0.014)$. According to spline fitting, the progression of vascular calcification was significantly increased after 45 years in smokers compared with that after 60 years in never smokers. Quitting smoking may compromise the deleteriousness of the vascellum especially in younger adults. However, the difference in age-related calcification among never smokers with or without secondhand smoke exposure was minor, regardless of the definition by selfreport, serum cotinine, or urinary 4-(methylnitrosamino)-1(3-pyridyl)-1-butanol.

Conclusions Smoking significantly accelerated the progression of age-related subclinical atherosclerosis. Early smoking cessation should be encouraged among
Strengths and limitations of this study

- This study involves a nationally representative sample of the adult population of the USA, suggesting an advantageous generalisation of the findings.

- Data were collected using standardised and validated protocols, which suggested favourable repeatability.

- This study evaluated the potential modification for age-related abdominal aortic calcification (AAC) by active smoking or secondhand smoke exposure, defined by self-reports or serum nicotine metabolites.

- This study was based on a cross-sectional design, which was less likely to conclude the causality.

- AAC measurements were only performed in participants aged 40 years or more. Whether smoking influences age-related vascular calcification in adults aged $<40$ years warrants further investigation.

young smokers. The effect of passive smoking exposure on arteriosclerosis should be assessed further.

\section{INTRODUCTION}

Cardiovascular disease (CVD) has been the leading cause of death worldwide since the early 20th century, imposing a heavy burden on public health and healthcare systems. ${ }^{1}$ Atherosclerosis has always been the primary pathological basis of CVD among the wide array of underlying cardiovascular lesions. ${ }^{2}$ Early identification of populations at high risk of subclinical atherosclerosis is important to prevent the progression of CVD. ${ }^{3}$ Abdominal aortic calcification (AAC) is considered a key hallmark of atherosclerosis and an independent predictor of subsequent cardiovascular morbidity and mortality. ${ }^{1} 4$ Evidence from previous studies suggests that severe $\mathrm{AAC}$ is associated with a higher risk of major cardiovascular events, thus reflecting a poor cardiovascular health status. ${ }^{5}$ 
Epidemiological studies have demonstrated that vascular calcification or atherosclerosis is a common physiological process associated with ageing. ${ }^{67}$ With the improvement in the primary and secondary preventions of cardiovascular events, the association between age and aortic calcification during the contemporary era remains unknown. Smoking is a well-established modifiable risk factor for atherosclerosis and CVD via several underlying pathways, ${ }^{8}$ and it has been a critical environmental hazard for public health. ${ }^{9}$ It is noteworthy that worldwide, approximately $11 \%$ of deaths related to CVD are attributed to smoking exposure ${ }^{10}$ Furthermore, secondhand smoke (SHS) exposure in non-smokers has also been associated with CVD and other health consequences. ${ }^{11}$ Although several studies have demonstrated the associations between ageing or smoking and atherosclerotic events, the evidence on the interactive effect between ageing and active or passive smoking in aortic calcification is limited. ${ }^{12}$ Clarifying this issue may provide new insights into the atherosclerotic process and identify the targeted population who could potentially benefit from smoking cessation education. In this context, this study tested the hypothesis that the association between age and AAC was modified by smoking exposure, including active and secondhand smoking, in a nationally representative sample of the middle-aged and elderly population.

\section{METHODS}

\section{Study population}

The data were extracted from the National Health and Nutrition Examination Surveys (NHANES) 2013-2014, an ongoing nationally representative-sampling, stratifiedsampling, multistage probability-sampling survey designed by the Centers for Disease Control and Prevention of the USA. The methods and protocols have been described in previous studies. ${ }^{13} 14$ There were 10175 individuals in NHANES 2013-2014, and 3815 of them were adults aged 40 years of age and above. Pregnant women were excluded from the study $(n=3)$. Dual-energy X-ray absorptiometry (DXA) scans were conducted for participants aged 40 years and above. Aortic calcification adjacent to the lumbar vertebrae L1-L4 was assessed. Participants without a scan $(n=482)$ or with image invalidity $(n=190)$ were excluded. All adults with eligible AAC scores were included in this study $(\mathrm{n}=3140) .{ }^{15}$ Detailed information is available on the website http://wwwcdcgov/nchs/ nhanes/irba98htm.

\section{AAC measurement}

AAC could be easily assessed on lateral spine DXA scans. ${ }^{16}$ The scores were calculated from a lateral scan of the lumbar spine (L1-L4 vertebrae) that was acquired using the Hologic Discovery model A densitometers (Hologic, Marlborough, Massachusetts, USA) and Apex software V.3.2. ${ }^{16}$ The radiation exposure from DXA for the lateral spine scan was extremely low $(<20 \mathrm{uSv})$. All scans were viewed using the Optasia SpinAnalyzer software.
The 24-point semiquantitative score of abdominal aortic calcification (AAC-24, Kauppila score) was used for the primary analysis, and the AAC-8 score (Schousboe score) was used for sensitivity analysis. ${ }^{16}$ Both methods have been validated with favourable sensitivity and specificity to reflect the severity of AAC. AAC-24 was calculated according to the length of calcification at the posterior and anterior aortic walls contiguous to the L1-L4 lumbar vertebrae. Treating lines across the middle of the intervertebral spaces as segment boundaries, we found that the abdominal aorta was divided into eight segments. AAC was scored from 0 to 3 according to calcification length in the aortic wall of each segment ( 0 point: no calcification, 1 point: $\leq 1 / 3$ arterial wall in each segment, 2 points: $1 / 3-2 / 3,3$ points: $>2 / 3)$. AAC- 8 is a simplified method derived from the AAC-24. AAC-8 in the anterior or posterior aortic walls in front of the L1-L4 was scored 0-4 (0 point: no calcification, 1 point: no more than the length of one vertebra, 2 points: no more than the length of two vertebrae, 3 points: no more than the length of three vertebrae and 4 points: more than the length of 3 vertebrae). Therefore, AAC- 8 score is less influenced by small calcifications but need more skilled technologists compared with AAC-24. DXA examinations were performed by trained and certified radiology technologists. Detailed protocols have been presented in previous studies. ${ }^{16}$

\section{Smoking exposure and cigarette biomarker assessment}

Smoking was defined as smoking at least 100 cigarettes during his or her life, ascertained through self-reports including both past and current cigarette smoking status. Tobacco metabolites, serum cotinine and urinary 4-(me thylnitrosamino)-1-(3-pyridyl)-1-butanol (NNAL) were considered as biomarkers of passive smoking exposures in non-smokers. ${ }^{17}$ Three definitions were used to identify individuals with SHS exposure, including self-report, urinary NNAL $\geq 0.001 \mathrm{ng} / \mathrm{mL}$, or serum cotinine level of $\geq 0.015 \mathrm{ng} / \mathrm{mL}$, as previously mentioned. ${ }^{18}$ Serum cotinine and urinary NNAL were determined by highperformance liquid chromatography (HPLC)-tandem mass spectrometry, with atmospheric pressure chemical ionisation or electrospray ionisation, respectively. The biosample was pretreated with methyl-D3-cotinine or 4-( methylnitrosoamino)-1-(3-pyridyl)-1-butanol-1,2', $3^{\prime}, 4^{\prime}, 5^{\prime}$, $6^{\prime}-13$ C6 as the internal standard. A C18 HPLC column was used to separate metabolites. The eluents from these injections were monitored using a mass spectrometer. The $\mathrm{m} / \mathrm{z} 80$ product ion from the $\mathrm{m} / \mathrm{z} 177$ quasi-molecular ion was used to quantify serum cotinine.

\section{Demographic, clinical and laboratory variables}

Data concerning age, sex, race/ethnicity, smoking status, physical activity, alcohol consumption and history of chronic diseases were collected using standardised questionnaires during personal interviews. ${ }^{13}$ Physical examinations were conducted at the Mobile Examination Centre (MEC) with standardised protocols. Peripheral blood 
Table 1 Characteristics of the study population in the National Health and Nutrition Examination Surveys 2013-2014

\begin{tabular}{|c|c|c|c|}
\hline Variable & Overall & Age $\leq 65$ years & Age $>65$ years \\
\hline Age (years) & 57.38 (56.75 to 58.01$)$ & 51.89 (51.39 to 52.39$)$ & 73.18 (72.72 to 73.65$)$ \\
\hline Male (\%) & 48.08 & 49.3 & 44.56 \\
\hline Hispanic-Mexican & 6.98 & 8.03 & 3.98 \\
\hline Other ethnicity & 11.89 & 13.0 & 8.69 \\
\hline Non-Hispanic black & 10.12 & 10.78 & 8.23 \\
\hline \multicolumn{4}{|l|}{ Smoking status (\%) } \\
\hline Never smoking & 53.98 & 54.38 & 52.84 \\
\hline Former smoker & 28.15 & 24.27 & 39.33 \\
\hline Current smoker & 17.87 & 21.35 & 7.831 \\
\hline Moderate & 32.75 & 31.67 & 35.86 \\
\hline Vigorous & 18.91 & 22.41 & 8.83 \\
\hline Alcohol intake (g) & 4.69 (4.01 to 5.36$)$ & 5.31 (4.45 to 6.17$)$ & 2.98 (2.39 to 3.56$)$ \\
\hline $\mathrm{BMI}\left(\mathrm{kg} / \mathrm{m}^{2}\right)$ & 28.54 (28.17 to 28.9$)$ & 28.7 (28.25 to 29.15$)$ & 28.07 (27.56 to 28.58$)$ \\
\hline Triglycerides (mmol/L) & 1.81 (1.73 to 1.89$)$ & 1.84 (1.74 to 1.94$)$ & 1.74 (1.63 to 1.85$)$ \\
\hline Total cholesterol (mmol/L) & 5.09 (5.04 to 5.13$)$ & 5.18 (5.12 to 5.23$)$ & 4.83 (4.70 to 4.96$)$ \\
\hline HDL-C (mmol/)L & $1.42(1.40$ to 1.43$)$ & 1.41 (1.38 to 1.43$)$ & 1.44 (1.39 to 1.48$)$ \\
\hline eGFR (mL/min/1.73 m²) & 84.28 (83.28 to 85.28$)$ & 89.49 (88.26 to 90.72$)$ & 69.47 (68.52 to 70.42$)$ \\
\hline $\mathrm{SBP}(\mathrm{mm} \mathrm{Hg})$ & $125.42(124.31$ to 126.53$)$ & 122.66 (121.43 to 123.89$)$ & $133.28(131.63$ to 134.92$)$ \\
\hline
\end{tabular}

Statistics of variables are presented as weighted proportion (\%) or means $(\mathrm{Cl} \%)$.

ACEI, ACE inhibitor; ARB, angiotensin II receptor antagonist; BMI, body mass index; DBP, diastolic blood pressure; eGFR, estimated glomerular filtration rate; HDL-C, high-density lipoprotein cholesterol; SBP, systolic blood pressure.

was collected from the MEC and analysed in the central laboratory, according to standardised procedures. Race/ ethnicity was categorised into four groups: non-Hispanic white, non-Hispanic black, Hispanic-Mexican and others. Physical activity was categorised as vigorous, moderate and less active as presented elsewhere. ${ }^{19}$ Drinking was assessed via questionnaires for the preceding year. One drink was defined as $12 \mathrm{oz}$ of beer, $4 \mathrm{oz}$ of wine or $1 \mathrm{oz}$ of liquor, each covering approximately $10 \mathrm{~g}$ of alcohol. ${ }^{19}$ Body mass index (BMI) was calculated as weight divided by height squared $\left(\mathrm{kg} / \mathrm{m}^{2}\right)$. Subjects' triglyceride (TG), total cholesterol (TC), high-density lipoprotein cholesterol (HDL-C) and serum creatinine levels were determined in morning fasting status. The estimated glomerular filtration rate (eGFR) was calculated using the Chronic Kidney Disease Epidemiology Collaboration. ${ }^{13}$ Hypertension was defined as a self-reported diagnosis by a doctor, with systolic blood pressure of $\geq 140 \mathrm{~mm} \mathrm{Hg}$ or a diastolic blood pressure of $\geq 90 \mathrm{~mm} \mathrm{Hg}$. Diabetes was defined as receiving antihyperglycaemic therapy or glycated haemoglobin (HbAlc) levels of $\geq 6.5 \%$. $^{19}$

\section{Statistical analysis}

Continuous and categorical variables are presented as means and percentages, weighted with primary sampling units, pseudo-strata and appropriate sampling weights to account for the complex sampling design. Crude and multivariable logistic or linear regression models were applied to assess the association between age and AAC scores. According to previous studies, ${ }^{16}$ severe AAC was defined as an AAC-24 score of $\geq 6$ points and subclinical AAC was defined as an AAC-24 score of $\geq 2$ points. Logistic regression analysis was used to calculate ORs as primary analysis. Two adjusted models were used in this study. Model 1 was adjusted for sex and race/ethnicity. Model 2 was additionally adjusted for BMI, smoking 
Table 2 Association between age and severe or subclinical AAC in all participants or subgroups by smoking

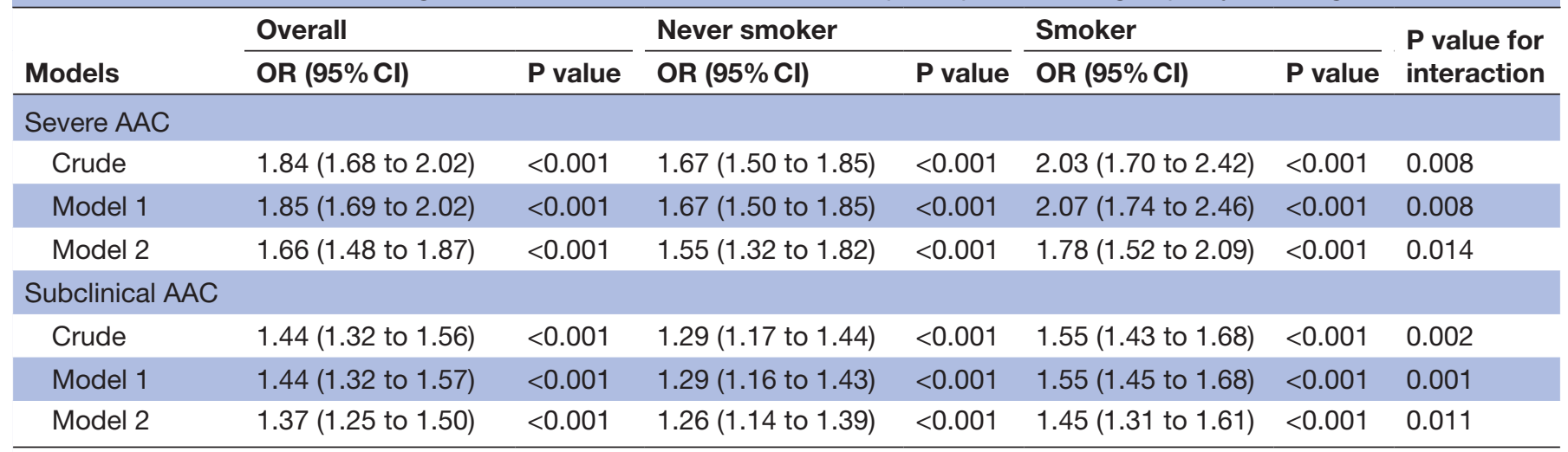

The AAC-24 (Kauppila score) was calculated according to the length of calcification at the posterior and anterior aortic walls contiguous to the L1-L4 lumbar vertebrae. Treating lines across the middle of the intervertebral spaces as segment boundaries, we divided the abdominal aorta into eight segments. AAC was scored from 0 to 3 according to calcification length in the aortic wall of each segment (0 point: no calcification; one point: $\leq 1 / 3$ arterial wall in each segment; 2 points: $1 / 3-2 / 3$; 3 points: $>2 / 3$ ). Severe AAC was defined as AAC-24 score $\geq 6$ points, and subclinical AAC was defined as AAC-24 scores $\geq 2$ points. The OR $(95 \% \mathrm{Cl})$ for the presence of severe or subclinical AAC was estimated using weighted logistic regression analysis. $\mathrm{P}$ value for interaction was used to assess the potential interaction between age and smoking for AAC using the survey-weighted Wald test. Model 1: adjusted for sex and race/ethnicity; model 2: additionally adjusted for body mass index, smoking status, alcohol consumption, physical activity, diabetes, hypertension, TC, TG, HDL-C, eGFR and decreasing lipid agents.

AAC-24, 24-point semiquantitative score of abdominal aortic calcification; AAC, abdominal aortic calcification; eGFR, estimated glomerular filtration rate; HDL-C, high-density lipoprotein cholesterol; TC, total cholesterol; TG, triglyceride.

status, alcohol consumption, physical activity, diabetes, hypertension, TC, HDL-C, eGFR, and decreasing lipid agents. The variance inflation factor of each variable was not more than 2.64, which did not suggest a multicollinearity of covariates. The survey-weighted Wald test was used to assess the potential interaction between age and smoking for AAC. The $\beta$ coefficient (95\% CI) was presented as the increased scores of AAC-24 or AAC-8 for each 5-year increase. Two-way fractional polynomial regression spline plots were used to visualise the relationship between age and vascular calcification among smokers or never smokers, and SHS or non-SHS. Statistical analysis was conducted with Stata V.SE15.0. All tests with a two-sided $p$ value of less than 0.05 were considered statistically significant.

\section{Patient and public involvement}

No patients were involved in the development of the research question or design of the study.

\section{RESULTS}

Overall, there were 3140 adults aged $\geq 40$ years with AAC scores in NHANES 2013-2014 (table 1). The weighted mean age was 57.4 years and $51.9 \%(\mathrm{n}=1622)$ were women. According to self-report, $54.0 \%$ were never smokers and $46.0 \%$ were smokers, including $28.2 \%$ ever smokers and $17.8 \%$ current smokers. Compared with participants aged $\leq 65$ years, older participants had higher proportions who were non-Hispanic white, quitting smoking, diabetes and cardiovascular medications, as well as less physical activity and lower renal function.
The association between age and AAC in all the participants, stratified by smoking, is shown in table 2 and online supplemental table 1. In univariable and sex-adjusted and race-adjusted logistic regression models, age was significantly associated with severe or subclinical ACC, with $85 \%$ and $44 \%$ increased odds for each 5-year increase in age (each $\mathrm{p}<0.001)$. After adjustment for sex, race, smoking, physical activity, alcohol intake, BMI, hypertension, diabetes, TC, TG, HDL-C, eGFR and decreasing-lipid agents, the association of age and severe or subclinical AAC remained significant with ORs of 1.66 (95\% CI 1.48 to 1.87 ) and 1.37 (95\% CI 1.25 to 1.50 ) for each 5-year increase in age $($ each $\mathrm{p}<0.001)$, respectively (table 2$)$. In addition, AAC-24 and AAC-8 scores were positively associated with age (online supplemental table 1). The association between age and AAC-24 score remained significant ( $\beta$ coefficient for each 5 -year increase in age: $0.43,95 \%$ CI 0.33 to $0.52, \mathrm{p}<0.001)$. Supplementary analysis for the association between smoking exposure and AAC was conducted (online supplemental tables 2-4). As expected, current smoking was significantly associated with AAC severity compared with never smokers. Treating current smokers as a reference, participants with smoking cessation of more than 10 years had lower AAC scores. However, the relationship between passive smoking and AAC was not observed.

Because smoking cigarettes are a strong risk factor for vascular injury, we assessed age-related aortic calcification in subgroups according to smoking status (table 2 and online supplemental table 1). As expected, the effect modification by smoking was observed ( $\mathrm{p}$ value for interaction $\leq 0.014)$. The OR for each 5-year increase in age 


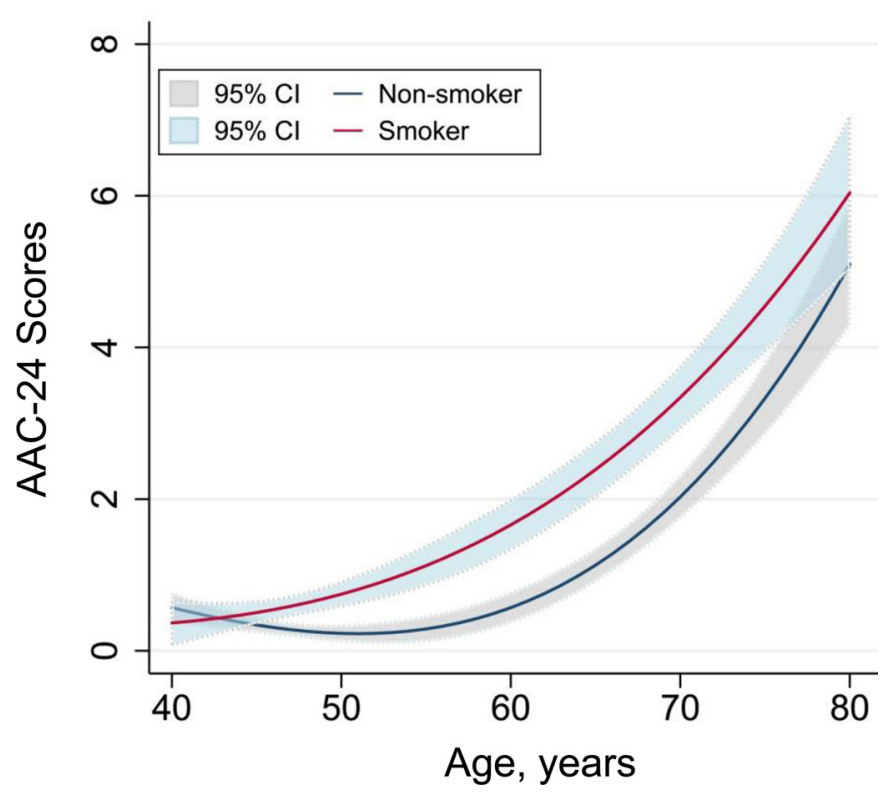

Figure 1 Spline fitting of age-related AAC in never smokers and smokers. The spline curve shows the relationship between scores of AAC-24 and age in smokers and never smokers based on fractional polynomial regression. The solid lines represent point estimates, and dashed lines represent $95 \%$ Cls. AAC-24 was calculated according to the length of calcification at the posterior and anterior aortic walls contiguous to the L1-L4 lumbar vertebrae. Treating lines across the middle of the intervertebral spaces as segment boundaries, we divided the abdominal aorta into eight segments. AAC was scored from 0 to 3 according to calcification length in the aortic wall of each segment ( 0 point: no calcification, 1 point: $\leq 1 / 3$ arterial wall in each segment, 2 points: $1 / 3-2 / 3$; 3 points: $>2 / 3$ ). Never smokers had a flat trend of age-related calcification before the age of 65 , whereas the calcification was significantly advanced after the age of 45 in subjects with smoking. AAC, abdominal aortic calcification; AAC-24, 24-point semiquantitative score of abdominal aortic calcification.

for severe AAC was 1.67 (95\% CI 1.50 to 1.85 ) among non-smoking participants vs 2.03 (95\% CI 1.70 to 2.42 ) among smoking participants. This suggests that the ever and current smoking may accelerate age-related AAC by $30 \%$ compared with never smoking status. Repeated analysis was performed using binary subclinical AAC and continuous AAC-24 and AAC-8 scores, and the correlations remained unchanged. According to spline fittings (figure 1), never smokers had a flat trend of age-related calcification before 65 years, whereas the calcification was significantly advanced after 45 years in smoking participants. Furthermore, compared with current smokers, participants who quit smoking had lower scores for agerelated calcification, especially for those aged 50-70 years (figure 2). However, among never smokers, SHS exposure did not significantly affect age-related AAC (figure 3).

\section{DISCUSSION}

In this nationally representative sample from the general population of the USA in 2013-2014, the association

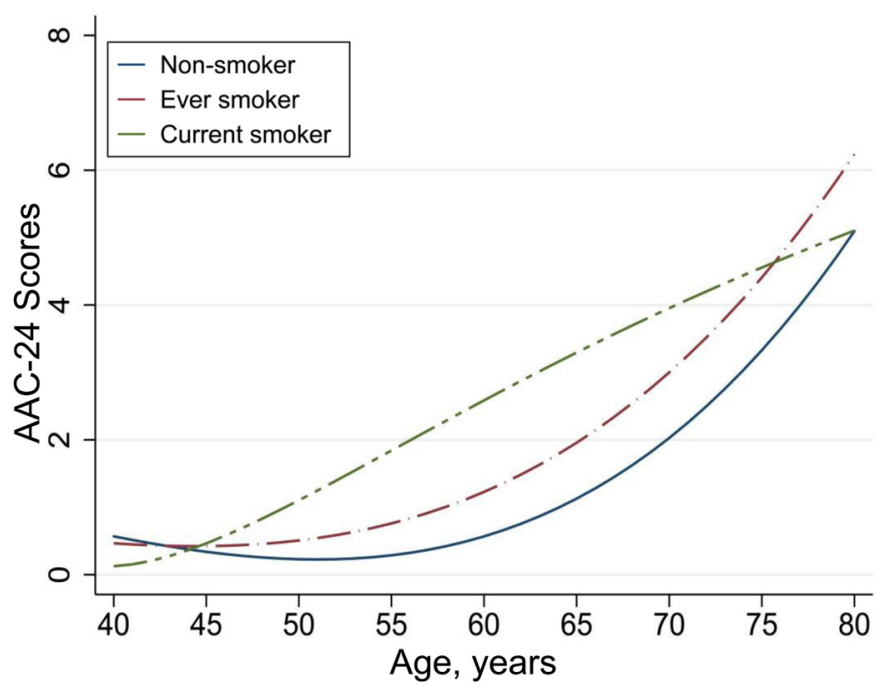

Figure 2 Visualisation of age-related AAC in smokers who have quit smoking or not. The spline curve shows the relationship between scores of AAC-24 and age in smokers who quit smoking or not based on fractional polynomial regression. The solid lines represent point estimates.

AAC, abdominal aortic calcification; AAC-24, 24-point semiquantitative score of abdominal aortic calcification.

between AAC and age differed, depending on the smoking status after adjustment for various confounders. Agerelated calcification was flat before 60 years old, whereas it was driven by smoking after 45 years old. Compared with persistent smoking, quitting smoking may mitigate the process of age-induced calcification, especially for adults aged 45-70 years. The deleterious effect of secondhand smoking on vascular calcification in adults was not observed in this study. Our study is of particular interest because our findings highlight the benefits of never smoking and early quit smoking in the process of age-related vascular calcification or atherosclerosis in the contemporary population.

Vascular calcification was considered to be a degenerative and end-stage consequence of the ageing process. ${ }^{6}$ Arterial calcification was detected in approximately onefourth of the populations aged 50 years. This proportion subsequently increased to beyond $60 \%$ in the population aged more than 75 years. ${ }^{7}$ Our results also consistently noted that AAC scores slowly increased in middle-aged participants, and robustly increased in the elderly participants aged $\geq 65$ years.

Vascular calcification has been linked with vascular injury and subclinical atherosclerotic disease. ${ }^{1620}$ Vascular wall calcification is a common byproduct of atherosclerotic plaques and was thus considered as a potential marker of subclinical atherosclerosis as well as a simple tool for cardiovascular risk stratification. ${ }^{16}$ Although previous reports have highlighted the implications of AAC as a biomarker of cardiovascular morbidity and mortality in adults, ${ }^{21-23}$ AAC has received less attention as a modifiable marker in preventing atherosclerosis and cardiovascular risk. ${ }^{24}$ Our findings suggest that quitting smoking is a simple and effective means to mitigate the process 


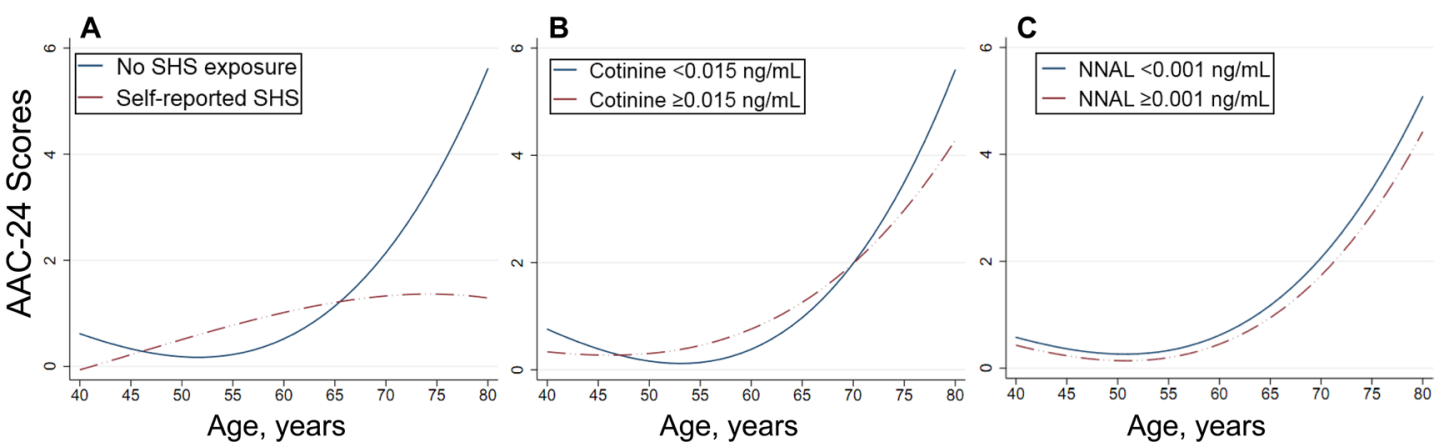

Figure 3 Age-related vascular calcification in never smoker with or without SHS exposure. Secondhand smoking exposure was defined by self-reports (A), serum cotinine (B) and urinary NNAL levels (C), respectively. The spline curve shows the relationship between AAC-24 scores and age in never smokers with or without SHS exposure based on fractional polynomial regression. AAC-24, 24-point semiquantitative score of abdominal aortic calcification; NNAL, 4-(methylnitrosamino)-1-(3pyridyl)-1-butanol; SHS, secondhand smoke.

of arterial calcification and atherosclerosis in the general population, especially in young adults. Lifestyle changes seem to be promising, low-cost and easy to spread.

Hisamatsu et al conducted an interesting study and demonstrated a positive association between smoking and subclinical atherosclerosis that was comprehensively assessed based on coronary artery calcification, carotid intima-media thickness and plaque, aortic artery calcification (from the aortic arch to iliac bifurcation) and ankle-brachial index. ${ }^{25}$ The relationship attenuated over the time of smoking cessation. Moreover, Pham et al supplemented the analysis for the relationship between smoking habits and the progression of coronary and aortic artery calcification in a follow-up study. ${ }^{26}$ These findings supported that smoking is a strong risk factor for coronary calcification and atherosclerosis. Jung and colleagues observed the positive association between AAC and long-term smoking exposure in 218 men aged 40-81 years. Even a low dosage of smoking increased the risk of AAC. ${ }^{27}$ Furthermore, Hirooka and colleagues analysed 313 Japanese and 302 Korean men aged 40-49 years and concluded that smoking with higher pack-years was significantly associated with artery calcium, suggesting a doseresponse pattern between cigarette use and progression of atherosclerosis. ${ }^{28} \mathrm{~A}$ small sample size and selection bias may limit the extrapolation. Our analysis also supported the increased risk of severe or subclinical angiosteosis associated with smoking. Notably, smoking cessation for more than 10 years could partly attenuate the harm of smoke exposure, which was consistent with the findings of previous reports. ${ }^{25} 2629$ Our results further emphasised that the association between age and ACC was modified by smoking and that the detrimental effects of ageing and smoking were not simply superposed.

Based on NHANES data, a recent study noted that serum cotinine levels, a biomarker of smoking, were associated with the risk of severe AAC in 2840 adults. ${ }^{16}$ However, circulating cotinine only reflects short-term exposure to smoking, and the interaction between smoking and age on the process of vascular calcification remains unknown. Our results highlighted that the process of age-related calcification was slow before 60 years of age. In contrast, smoking, especially persistent smoking, significantly accelerated this process since 40 years of age. Quitting smoking at an early stage may at least partially moderate the speed for adults aged $\leq 70$ years. To the best of our knowledge, our study is the first study that specifically investigates the interaction between age and smoking on the severity of aortic calcification in humans. Notably, previous studies have established the association between smoking and vascular calcification, suggesting the potential benefits of quitting smoking to prevent atherosclerosis. ${ }^{8} 163031$ However, the complex interaction with the ageing process needs to be clarified, which may provide new insights into the optimal time to prevent atherosclerosis by tobacco control. ${ }^{30}$ Our results suggest small benefits of quit smoking in the elderly aged $>70$ years, which highlights the importance of early action to reduce the burden of vascular calcification.

SHS exposure has been proven to be associated with CVD and adverse outcomes. ${ }^{11}$ Approximately $85 \%$ of SHS was derived from side-stream smoke that seemed potentially more harmful due to non-filtering. ${ }^{12}$ As another purpose, we investigated the relationship between AAC and SHS based on self-reports, and serum cotinine or urine NNAL in never smokers. However, the neutral link between arterial calcification and passive smoking that we noted might be not the ultimate conclusion. The questionnaires of secondhand smoking were only designed for the preceding 7 days, although we used the definition of potentially persistent SHS exposure in indoor settings at job or home. Serum cotinine and urinary NNAL, as more accurate biomarkers of smoking exposure than selfreports, have been validated to estimate the SHS exposure in the past 2-4days and 6-12 weeks, respectively. ${ }^{18}$ The relationship was also insignificant in stratifications by serum cotinine or urinary NNAL. Both biomarkers only reflected exposures over the past several days or weeks, which were insufficient to reflect long-term exposure. The progression of vascular calcification is a chronic process, even beyond the years. Therefore, further studies may need multiple repeated measurements or questionnaires 
over the years to assess the effect of SHS on age-related calcification.

\section{Limitations and strengths}

Our study has some strengths that support favourable repeatability, including a nationally representative sample of the adult population, standardised and validated protocols, and full adjustment for a large number of potential confounders. However, our conclusions should be explained in consideration of the following limitations. First, this study was based on a cross-sectional design, which was less likely to conclude the causality. More convincing evidence could be obtained from a longitudinal study with multiple measurements. Second, the analysis of the relationship between SHS and AAC was not the ultimate seal. The duration of SHS exposure should be considered in future studies. Third, AAC measurement was performed only in the population aged 40 years or above. Whether smoking influences age-related vascular calcification in adults aged $<40$ years old warrants investigation. Fourth, residual confounding factors could not be eliminated, although we strictly adjusted for 16 covariates in the regression model.

\section{CONCLUSIONS}

Our results indicate that smoking significantly modifies the association between age and AAC severity in adults aged 40 years or above. Age-related vascular calcification is accelerated by smoking, which is partly reduced by smoking cessation. However, whether SHS affects this process requires further investigation.

Acknowledgements The authors thank all participants and staff in National Health and Nutrition Examination Surveys for their great contributions.

Contributors $Y Y$ and $X Y$ contributed to the conceptualisation and developed the protocols. LL and SW organised and analysed all data. XY was responsible for the project administration. All authors wrote and reviewed the manuscript, and approved the final manuscript. YY and $X Y$ accepted full responsibility for the finished work and the conduct of the study, had access to the data, and controlled the decision to publish.

Funding The authors have not declared a specific grant for this research from any funding agency in the public, commercial or not-for-profit sectors.

Competing interests None declared.

Patient consent for publication Consent obtained directly from patient(s).

Ethics approval The protocols and procedures of the National Health and Nutrition Examination Surveys(NHANES) study were agreed by the Research Ethics Review Board of the Centers for Disease Control and Prevention of the USA (protocol number. 2011-17). This study was conducted in accordance with the Declaration of Helsinki. The protocols of the NHANES were approved by the Ethics Review Board of the National Center for Health Statistics. All participants provided informed consent.

Provenance and peer review Not commissioned; externally peer reviewed.

Data availability statement All data came from the NHANES study (http://www. cdc.gov/nchs/nhanes). Data are available on reasonable request from the CDC of US or corresponding authors.

Supplemental material This content has been supplied by the author(s). It has not been vetted by BMJ Publishing Group Limited (BMJ) and may not have been peer-reviewed. Any opinions or recommendations discussed are solely those of the author(s) and are not endorsed by BMJ. BMJ disclaims all liability and responsibility arising from any reliance placed on the content. Where the content includes any translated material, BMJ does not warrant the accuracy and reliability of the translations (including but not limited to local regulations, clinical guidelines, terminology, drug names and drug dosages), and is not responsible for any error and/or omissions arising from translation and adaptation or otherwise.

Open access This is an open access article distributed in accordance with the Creative Commons Attribution 4.0 Unported (CC BY 4.0) license, which permits others to copy, redistribute, remix, transform and build upon this work for any purpose, provided the original work is properly cited, a link to the licence is given, and indication of whether changes were made. See: https://creativecommons.org/ licenses/by/4.0/.

ORCID iD

Xiongli Yue http://orcid.org/0000-0002-2937-6824

\section{REFERENCES}

1 Li Y-W, Chen W-L. Clinical relevance of serum uric acid and abdominal aortic-calcification in a national survey. Clin Cardiol 2020;43:1194-1201.

2 Lusis AJ. Atherosclerosis. Nature 2000;407:233-41.

3 Kälsch H, Lehmann N, Moebus S, et al. Aortic calcification onset and progression: association with the development of coronary atherosclerosis. J Am Heart Assoc 2017;6 doi:10.1161/ JAHA.116.005093

4 Wilson PW, Kauppila LI, O'Donnell CJ, et al. Abdominal aortic calcific deposits are an important predictor of vascular morbidity and mortality. Circulation 2001;103:1529-34.

5 Szulc P. Abdominal aortic calcification: a reappraisal of epidemiological and pathophysiological data. Bone 2016;84:25-37.

6 Zhang B, Miller VM, Miller JD. Influences of sex and estrogen in arterial and valvular calcification. Front Endocrinol 2019;10:622.

7 Cannata-Andía JB, Rodríguez-García M, Carrillo-López N, et al. Vascular calcifications: pathogenesis, management, and impact on clinical outcomes. J Am Soc Nephrol 2006;17:S267-73.

8 Wang Z, Wang D, Wang Y. Cigarette smoking and adipose tissue: the emerging role in progression of atherosclerosis. Mediators Inflamm 2017;2017:3102737

9 GBD 2015 Tobacco Collaborators. Smoking prevalence and attributable disease burden in 195 countries and territories, 19902015: a systematic analysis from the global burden of disease study 2015. Lancet 2017;389:1885-906.

10 Ezzati M, Henley SJ, Thun MJ, et al. Role of smoking in global and regional cardiovascular mortality. Circulation 2005;112:489-97.

11 Asfar T, Koru-Sengul T, Ruano-Herreria EC, et al. Secondhand smoke exposure among high-risk patients in the United States (NHANES 2001-2012): implications for clinical practice. Nicotine Tob Res 2019;21:551-6.

12 Park YS, Lee C-H, Kim Y-I, et al. Association between secondhand smoke exposure and hypertension in never smokers: a crosssectional survey using data from Korean National health and nutritional examination survey V, 2010-2012. BMJ Open 2018;8:e21217.

13 Wang S, Liu Y, Liu J, et al. Mitochondria-derived methylmalonic acid, a surrogate biomarker of mitochondrial dysfunction and oxidative stress, predicts all-cause and cardiovascular mortality in the general population. Redox Biol 2020;37:101741.

14 Rossato LT, de Branco FMS, Azeredo CM, et al. Association between omega-3 fatty acids intake and muscle strength in older adults: a study from national health and nutrition examination survey (NHANES) 1999-2002. Clin Nutr 2020;39:3434-41.

15 Kathryn Porter. Data from: the National health and nutrition examination survey (NHANES). 2013-2014. The centers for disease control and prevention of United States, September 30, 2018. Available: https://www.cdc.gov/nchs/nhanes/

16 Wen X, Xia Y, Guo M, et al. Association between serum cotinine and severe abdominal aortic calcification in US adults. Angiology 2020;71:333-9.

17 Fu Z, Jiang H, Xu Z, et al. Objective secondhand smoke exposure in chronic obstructive pulmonary disease patients without active smoking: the U.S. National health and nutrition examination survey (NHANES) 2007-2012. Ann Transl Med 2020;8:445. doi:10.21037/ atm.2020.03.145

18 Fu Z, Jiang H, Xu Z, et al. Objective secondhand smoke exposure in chronic obstructive pulmonary disease patients without active smoking: the U.S. National health and nutrition examination survey (NHANES) 2007-2012. Ann Transl Med 2020;8:445. doi:10.21037/ atm.2020.03.145

19 Wang S, Tian W, Liu Y, et al. Temporal trend of circulating trans-fatty acids and risk of long-term mortality in general population. Clin Nutr 2021;40:1095-101. 
20 Budoff MJ, Achenbach S, Blumenthal RS, et al. Assessment of coronary artery disease by cardiac computed tomography: a scientific statement from the American heart association Committee on cardiovascular imaging and intervention, Council on cardiovascular radiology and intervention, and Committee on cardiac imaging, Council on clinical cardiology. Circulation 2006;114:1761-91.

21 Bastos Gonçalves F, Voûte MT, Hoeks SE, et al. Calcification of the abdominal aorta as an independent predictor of cardiovascular events: a meta-analysis. Heart 2012;98:988-94.

22 Martino F, Di Loreto P, Giacomini D, et al. Abdominal aortic calcification is an independent predictor of cardiovascular events in peritoneal dialysis patients. Ther Apher Dial 2013;17:448-53.

23 Bolland MJ, Wang TKM, van Pelt NC, et al. Abdominal aortic calcification on vertebral morphometry images predicts incident myocardial infarction. J Bone Miner Res 2010;25:505-12.

24 Golledge J. Abdominal aortic calcification: clinical significance, mechanisms and therapies. Curr Pharm Des 2014;20:5834-8.

25 Hisamatsu T, Miura K, Arima $\mathrm{H}$, et al. Smoking, smoking cessation, and measures of subclinical atherosclerosis in multiple vascular beds in Japanese men. J Am Heart Assoc 2016;5. doi:10.1161/ JAHA.116.003738. [Epub ahead of print: 2908 2016]

26 Pham T, Fujiyoshi A, Hisamatsu T, et al. Smoking habits and progression of coronary and aortic artery calcification: a 5-year follow-up of community-dwelling Japanese men. Int $J$ Cardiol 2020;314:89-94.

27 Jung J-G, Wu L-T, Kim J-S, et al. Relationship between smoking and abdominal aorta calcification on computed tomography. Korean $\mathrm{J}$ Fam Med 2019;40:248-53.

28 Hirooka N, Kadowaki T, Sekikawa A, et al. Influence of cigarette smoking on coronary artery and aortic calcium among random samples from populations of middle-aged Japanese and Korean men. J Epidemiol Community Health 2013;67:119-24.

29 McEvoy JW, Nasir K, DeFilippis AP, et al. Relationship of cigarette smoking with inflammation and subclinical vascular disease: the multi-ethnic study of atherosclerosis. Arterioscler Thromb Vasc Biol 2015;35:1002-10.

30 Lehmann N, Möhlenkamp S, Mahabadi AA, et al. Effect of smoking and other traditional risk factors on the onset of coronary artery calcification: results of the Heinz Nixdorf recall study. Atherosclerosis 2014;232:339-45.

31 Rasmussen T, Frestad D, Køber L, et al. Development and progression of coronary artery calcification in long-term smokers: adverse effects of continued smoking. J Am Coll Cardiol 2013;62:255-7. 